\title{
Decentralizing Privacy Enforcement for Internet of Things Smart Objects
}

\author{
Gokhan Sagirlar,Barbara Carminati,and Elena Ferrari \\ DISTA, University of Insubria, Italy.
}

\begin{abstract}
Internet of Things (IoT) is now evolving into a loosely coupled, decentralized system of cooperating smart objects, where highspeed data processing, analytics and shorter response times are becoming more necessary than ever. Such decentralization has a great impact on the way personal information generated and consumed by smart objects should be protected, because, without centralized data management, it is more difficult to control how data are combined and used by smart objects. To cope with this issue, in this paper, we propose a framework where users of smart objects can specify their privacy preferences. Compliance check of user individual privacy preferences is performed directly by smart objects. Moreover, acknowledging that embedding the enforcement mechanism into smart objects implies some overhead, we have extensively tested the proposed framework on different scenarios, and the obtained results show the feasibility of our approach.
\end{abstract}

Keywords: Internet of Things (IoT), Smart Objects, Privacy.

\section{Introduction}

Internet of Things (IoT) technologies are revolutionizing our daily lives [1], building around us a pervasive environment of smart objects able, not only to sense data, but also to interact with other objects and to aggregate data sensed through different sensors. This allows smart objects to locally create new knowledge, that could be used to make decisions, such as quickly trigger actions on environments, if needed. Smart objects are very heterogeneous in terms of data sensing and data processing capabilities. Some of them can only sense data, others can perform basic or complex operations on them. Such a scenario enacts the transition from the Internet of Things to the Internet of Everything, a new definition of IoT seen as a loosely coupled, decentralized system of cooperating smart objects, which leverages on alternative architectural patterns with regards to the centralized cloud-based one, such as fog computing. Such a trend towards decentralization reduces the amount of data that is transferred to the cloud for processing and analysis, and can also be instrumental to improve security and privacy of the managed data, a major concern in the IoT scenario. However, decentralization, if not properly governed, might also imply loss of control over the data, with consequences on individual privacy.

In this paper, we focus on the challenging issue of designing a decentralized privacy enforcement mechanism, where compliance check of user individual privacy preferences is performed directly by smart objects, rather than by a central entity. Restrictions on devices' capabilities let us discard existing proposals for decentralized access control (e.g., [2, 3, 4, 5]), as these

\footnotetext{
*E-mail addresses:

Gokhan Sagirlar: gsagirlar@uninsubria.it

Barbara Carminati: barbara.carminati@uninsubria.it

Elena Ferrari: elena.ferrari@uninsubria.it
}

heavily rely on cryptographic primitives. Previously, in [6], we addressed the problem of specifying and enforcing privacy preferences in the IoT scenario, but for a centralized architecture, that is, a scenario where devices have only the capability to sense data and send them to a data center for being analyzed. In this setting, the enforcement monitor analyzes every consumer query and decides if the privacy policy of the consumer satisfies the privacy preferences specified by owners of devices generating the data. Compared to this approach, decentralized privacy enforcement scenario requires to address several new important research challenges, as smart objects are characterized by heterogeneous processing capabilities. To address these challenges, in this paper, we extend the privacy preference model proposed in [6], by designing a set of privacy meta-data that are used by smart objects for locally checking privacy preferences and for locally enforcing user privacy preferences at smart object level. Smart objects are thus able to derive privacy meta-data for newly created data items, keep track of the operations performed over data items, denoted as history, in order to ease privacy preference enforcement, and, finally, check compliance of the privacy policy of the data consumer with the privacy preferences of data items. To the best of our knowledge this is the first work proposing a decentralized enforcement of privacy preferences able to work locally at smart object level.

Acknowledging that embedding the enforcement mechanism into smart objects might imply some overhead, we have extensively tested the proposed framework. In doing the experiments, we have considered several scenarios, by varying the complexity of the privacy preferences, smart object networks, and evaluated queries. The experiments allow us to asses the feasibility of the proposed approach in a variety of application domains.

The remainder of this paper is organized as follows. Section 
2 describes the system model and design assumptions of the proposed privacy preserving framework. Section 3 introduces the privacy preference model. Section 4 presents the proposed enforcement mechanism. Experimental results are illustrated in Section 5 Section 6discusses related work, whereas Section 7 concludes the paper.

\section{System Model and Assumptions}

The main purpose of the framework is to enhance the privacy of IoT users making them able to set and enforce their privacy preferences. The privacy enforcement mechanism relies on some privacy meta-data (encoding user privacy preferences, data categories, and data history), generated by smart objects. Data generated by smart objects are modelled as data streams. Moreover, we assume that smart objects are able to perform a set of SQL-like operations (i.e., projection (П), aggregation $(\Sigma)$, join $(\bowtie)$ and selection $(\sigma)$ ) over data streams, as SQL represents the query model adopted by many data processing systems in the IoT domain [7]. These SQL operators are continuously evaluated on every incoming tuple, whereas operations like join and aggregation are processed on groups of tuples organized as sliding windows. In the following, we present the system model and our design assumptions.

\subsection{System model}

We consider a decentralized IoT system consisting of the following entities:

Subjects. We consider two types of subjects, namely data owners and data consumers. Data owner is the user that owns an IoT smart object. We assume that data owners define individual privacy preferences over their data (cfr. Section 3 for a detailed discussion) in order to control how their data are distributed and processed. In contrast, data consumer consume raw or processed data from IoT systems. We assume that data consumers adopt privacy practices that specify how the consumer will use the users' data.

Smart Objects. Smart objects are able to sense, process and aggregate data, and interact with other smart objects. However, they are extremely heterogeneous regarding their capabilities and roles in the IoT ecosystem and this also impacts the privacy checks they can perform. In particular, we need to classify smart objects based on their capabilities so as to assign to each of them a possible role, and corresponding function in the proposed decentralized privacy preference enforcement mechanism. On the basis of several standardization recommendations from different organizations [8, 9], we propose a smart objects taxonomy based on the object smartness, aka abilities in sensing and processing individual data, as follows:

- First Level of Smartness: at this level, devices have a very limited capacity, being able only to act as basic sensors, for sensing data from the environment.

- Second Level of Smartness: at the second level, devices have a limited capacity and computing power, making them only able to perform basic operations by their own, like projection and selection, which do not require window-based processes that are working on tuples organized as sliding windows, such as join or aggregation. These devices might be virtual or physically manufactured, e.g., a field-programmable gate array (FPGA) or hard-coded devices.

- Third Level of Smartness: at this level, we group those devices that have enough capacity and computing power for performing complex operations by their own, including windowbased operations.

According to this taxonomy, a smart object ( $S O$ ) may play three different roles in the proposed decentralized privacy enforcement 1

- Sensing Smart Objects: the main objective of this type of smart objects is sensing data from the environment. Typically, a smart object with a first level of smartness will play this role.

- Processor Smart Objects: this type of smart objects aim at creating new information from sensed data by performing operations over them. Complexity of these operations depends on the smartness level of the object. As such, a smart object with second or third level of smartness will be able to play this role.

- Consumer Smart Objects: a smart object playing this role is in charge of verifying user privacy preferences before sending data to consumers. This process is called privacy preference compliance (see Section 4.3 for more details). Such role can be taken by SOs with third level of smartness (e.g., gateway devices, such as Dell Edge Gateways or HPE Edgeline Gateways).

Example 1 Throughout the paper, we will use as an example a smart home system. We assume that the smart home is equipped with a smart heating and electricity usage control systems. The smart heating system is built on top of a network of smart objects able to sense temperature values from several rooms, adjust temperature, if needed, and share data with the heating company. The smart electricity usage system relies on a network of smart objects able to sense electricity usage data, locally compute usage trends, and send them to the electricity distribution company (see Figure 1). In this scenario, sensing smart objects are those devices used for measuring electricity usage and sensing room temperature, whereas processor smart objects are those used to locally aggregate data sensed by sensing SOs, with the purpose of generating trends of electricity usage as well as average rooms temperature. Finally, smart objects sending collected information to electricity and heating system companies can be categorized as consumer smart objects, as their main role is to send data to consumers.

\subsection{Security assumptions}

Security vulnerabilities, if not properly addressed and prevented, may allow adversaries to damage correct execution of IoT systems and the proposed privacy preserving mechanism. In designing the privacy preserving mechanism, we have assumed that smart objects conduct a set of defence strategies to protect the IoT system from security threats and attacks. Let

\footnotetext{
${ }^{1}$ We postpone the reader to Section 4 for details about processes and algorithms implemented for each role.
} 


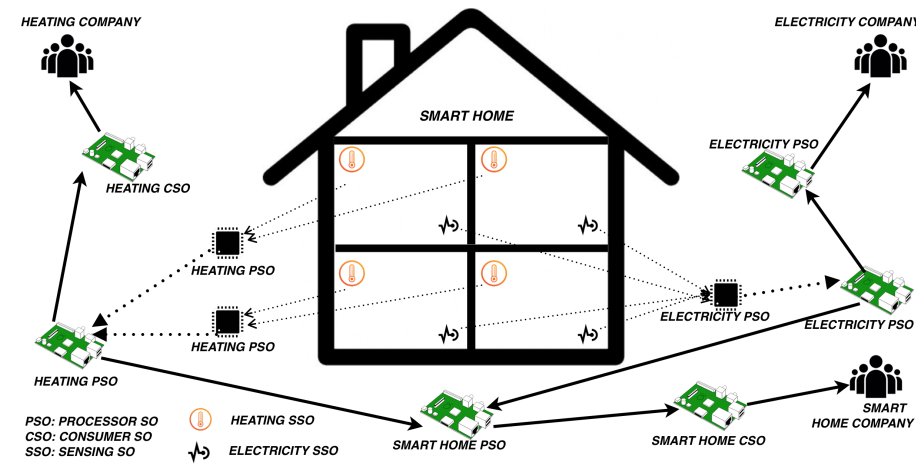

Figure 1: Smart home scenario

us first define the adversary model. We assume that an adversary is capable to block and intercept communication, and is also able to overhear, inject and delete messages exchanged between smart objects, as well as analyze communication patterns. Moreover, we assume that sensing and processor SOs are susceptible to be compromised by adversaries and thus can act maliciously, whereas consumer SOs are honest, i.e., they behave as expected according to the proposed algorithms. This assumption is based on the conjecture that IoT gateway devices, such as Dell Edge Gateways ${ }^{2}$ or HPE Edgeline Gateways ${ }^{3}$ hosting consumer SOs are securely produced by their manufacturers and have high resources. Indeed, as stated by [10], a vulnerability in the hardware of such devices will inevitably threat the whole system. Therefore, we assume that device manufactures will do their best in designing secure gateway devices. Given the adversary model, the privacy preserving mechanism relies on the following defense techniques that are assumed to be conducted by smart objects:

Network monitoring and intrusion detection systems. We assume that consumer SOs are complemented with network monitoring (e.g., [11]) and intrusion-detection mechanisms (e.g., [12]). Because such mechanisms help to detect and reduce effects of Denial of Service (DoS) and node compromise attacks. Indeed, as our approach relies on machine to machine communication among smart objects in which the operations of one smart object, e.g., meta-data generation and update, mostly depend on the data sent by another smart object, DoS attacks may affect the correct execution of the protocols. Furthermore, compromised and malicious processor SOs can alter the derivation of new privacy meta-data with the result of not correctly enforcing privacy requirements of the data owners. By virtue of equipping consumer SOs with intrusion detection mechanisms, we assume that consumer SOs analyze the network and detect compromised and infected devices acting maliciously or violating policies.

Lightweight encryption and authentication mechanisms. We also assume that all smart objects are equipped with encryption mechanisms (such as [13, 14]) that make use of lightweight cryptographic primitives (e.g., hash, nonce, etc), and thus able

\footnotetext{
${ }^{2}$ dell.com/us/business/p/edge - gateway

${ }^{3}$ hpe.com/emea $a_{e}$ urope/en/servers/edgeline - iot - systems
}

to encrypt the data and the meta-data before sending them. In such a way, that data cannot be disclosed and privacy meta-data cannot be compromised during the communication.

We also assume that smart objects are equipped with hop by hop authentication protocols, such as [15]. This is a a way to contrast impersonation attacks, where an adversary might aim to seize the identity of a legitimate smart object, such as access credentials of the device, to act on behalf of the legitimate device (e.g. injecting fake data) that may compromising privacy preference enforcement.

Clone detection protocols. We assume that smart objects are equipped with a globally aware distributed clone detection protocol (such as [16]). Cloned smart objects may generate fake data and affect the correct execution of the privacy protection protocols, as well as they can be used to disable and compromise honest smart objects and thus damage privacy enforcement.

\section{The Privacy Model}

In this section, we present the privacy preference model and an approach to automatically generate privacy preferences for new generated data.

\subsection{Privacy Preferences}

Privacy preferences are specified according to the model proposed in [6], which has been designed to cope with two relevant challenges that IoT ecosystems pose on the management of individual privacy.

The first is that, due to the complexity of the data flows among IoT devices, users easily lose the control on how their data are distributed and processed. This is even made worse considered that data generated by different IoT devices can be fused to infer new information on individuals. To give individuals more control, we support privacy preferences that are able to specify additional constraints with regards to those that can be expressed by standard privacy languages. In particular, our privacy preferences make a user able to pose conditions not only on which portion can be collected, for which usage purpose, for how long, and by whom (like traditional privacy policies and preferences), but also make him/her able to pose limitations on how data can be used to derive new information (e.g., by explicitly stating what cannot be derived from users data by analytics processes).

The second issue the privacy model considers is related to the management of new derived data. Indeed, although privacy preferences help individuals at regulating the derivation of new data, they do not regulate how these new data will be managed. At this purpose, the privacy model implements a strategy for the automatic definition of additional privacy preferences for newly derived data. The new preference is defined by taking into account the privacy preferences associated with each single piece of data used for computing the new derived data.

Similar to other privacy preference models, we organize both purposes and data categories into trees. This simplifies the specification of privacy preferences, in that a preference stated 
for a node in the tree representing a purpose/category (e.g., the physical state data category) implicitly holds also for all nodes in its subtree (e.g., heart beat, blood pressure, etc.). Moreover, besides making individual able to specify the purposes for which is allowed the data usage, the model aims at making him also able to pose limitation. As such, the privacy preference allows the specification of those purposes for which the access is explicitly prohibited. This is represented via an element, called intended purposes - ip, which is modeled as a pair (Aip, Exc), with the following semantics: all requests with a purpose that descends from elements in Aip are authorized, except for those that descend from any element in Exc in the purpose tree.

Besides intended purposes, privacy preferences allow users to explicitly state which portions of their personal data can be combined/aggregated with other data. Such requirements are expressed in terms of allowed/denied data categories, modelled through the so called joint access constraints - jac. More formally, a joint access constraint for an attribute $a$ is a tuple $\langle A d c$, $E x c$, $i p\rangle$, where $A d c$ is the set of data categories that can be jointly accessed with $a, E x c$ is a set of data categories that specialize elements in $A d c$ and that cannot be jointly accessed, whereas ip is an intended purpose. This constraint specifies that the categories that descend from those specified in $A d c$ can be jointly accessed with attribute $a$ for the purposes authorized by ip, except for those that descend from any element in Exc. Finally, a privacy preference allows data owners to specify what cannot be derived from their data by the analytics processes. This is specified through a category derivation constraint $-c d c$, that specifies a set of data categories, denoted as $C d c$, that cannot be derived using the data 4

A privacy preference is therefore formally defined as follows:

Definition 1. (Privacy preference) A privacy preference pp is a tuple $\langle\alpha$, consumer, ip, jac, $c d c\rangle$, where $\alpha$ is an attribute of a data stream generated by a smart object, consumer specifies the set of data consumers authorized to access $\alpha$, ip specifies the intended purposes for which $\alpha$ can be collected and used, jac specifies a joint access constraint, whereas cdc is category derivation constraint specifying a set of data categories which cannot be derived from $\alpha$.

A summary of the notations related to privacy preferences is given in Table 1 .

\begin{tabular}{|c|c|}
\hline Element & Abbreviation \\
\hline Consumer ids & consumer \\
\hline Intended Purpose & ip \\
\hline Allowed intended purposes of ip & ip.Aip \\
\hline Prohibited intended purposes of ip & ip.Exc \\
\hline Joint access constraint & jac \\
\hline Allowed data categories of jac & jac.Adc \\
\hline Prohibited data categories of jac & jac.Exc \\
\hline Intended purposes for jac & jac.ip \\
\hline Category derivation constraint & $c d c$ \\
\hline
\end{tabular}

Table 1: Privacy preference's notation

\footnotetext{
${ }^{4}$ In what follows, we will use the dot notation to refer to specific components
} of jac and ip elements.
Example 2 Let us consider the smart home scenario given in Example 1 and let us suppose that a user, say Charlotte, has installed this smart home system in her apartment and that she wants to specify a privacy preference $p p$ for the temperature data sensed by the system. In particular, Charlotte wants to share her data only with Smart Home Company and she wants to release the sensed data only for the administration of the heating system. Moreover, she allows temperature data to be joined with generic information, as well as health data of her smart watch (e.g., body temperature) as this could help her to properly customize room temperature. Thus, she agrees that temperature data is joined with generic information and health data for administration purpose. However, she prefers that from these data it is not possible to derive sensitive information, such as her current location in the apartment or the number of persons in the room. To model these requirements, Charlotte can specify pp as: 〈temperature, \{smart-home-company\}, $\langle\{$ admin\}, $\emptyset\rangle$, $\langle\{$ generic, health\},,$\langle\{$ admin $\}, \emptyset\rangle\rangle$, \{sensitive $\}\rangle$.

As a final note, it is important to highlight that the additional elements that we have introduced to cope with the new issues that IoT scenario poses, (i.e., consumer identities, joint access constraints, and category derivation constraints), are not mandatory. This provides flexibility to vendors to decide whether to adhere only to common privacy practices (e.g., supporting privacy preferences with purpose, retention, and third party elements) or to exploit the extended privacy preferences so as to provide users with a more complete control on their personal data.

\subsection{Privacy Preference Derivation}

An interesting feature of the model in [6] is its ability to associate privacy preferences with new data generated by smart objects. These new privacy preferences are defined by combining the privacy preferences specified by each owner of smart objects sensing the data involved in the derivation process. Privacy preferences are combined by taking the most conservative approach so that the resulting privacy preference satisfies the constraints specified in all the privacy preferences associated with smart objects involved in the data fusion process. More precisely, the composed privacy preference is defined by taking the intersection of the consumer, jac.Adc and ip.Aip elements, and the union of the jac.Exc, ip.Exc and $c d c$ fields of the privacy preferences involved in data fusion. Formally a composed privacy preference is defined as follows:

Definition 2. (Composed privacy preference) Let $\alpha$ be an attribute belonging to the output stream generated by a smart object, and let At be the set of attributes from which $\alpha$ is derived. The privacy preference $p p=\langle\alpha$, consumer, ip, jac, $c d c\rangle$ for $\alpha$ is derived as follows:

- consumer is defined as the intersection of the ids in the consumer field with all privacy preferences associated to all attributes in At, that is: consumer = $\bigcap_{a \in A t}$ a.pp.consumer

- The intended purpose component ip is defined as: 
- Aip (allowed intended purposes) is the intersection of the purposes implied by the ip's allowed intended purposes of all attributes in At, that is: Aip $=\bigcap_{a \in A t} \cup_{p \in a . p p . i p . A i p} p^{\downarrow}$, where $p^{\downarrow}$ denotes a set composed of $p$ and all purposes descending from $p$ in the purpose tree.

- Exc (prohibited purposes) is the union of the ip's prohibited purposes of the privacy preferences specified for At's attributes, that is: Exc $=\bigcup_{a \in A t}$ a.pp.ip.Exc

- The joint access constraint jac is defined as: - Adc (allowed data categories) is the intersection of the jac's allowed data categories of all attributes in At, that is: Adc = $\bigcap_{a \in A t} \bigcup_{c \in a . p p . j a c . A d c} c^{\downarrow}$, where $c^{\downarrow}$ denotes the set composed of $c$ and all categories that descend from $c$ in the data category tree.

- Exc (exception) is the union of the jac's exceptions specified for all attributes in At, that is:

Exc $=\bigcup_{a \in A t}$ a.pp.jac.Exc;

- ip (jac intended purposes) is defined following the same criteria of the preference intended purposes, that is: ip.Aip $=\bigcap_{a \in A t} \cup_{p \in a . p p . j a c . i p . A i p} p^{\downarrow}$, and ip.Exc = $\bigcup_{a \in A t}$ a.pp.jac.ip.Exc.

- The prohibited data categories cdc are defined as the union of prohibited data categories specified in the privacy preferences of all attributes in At, that is: $c d c=$ $\bigcup_{a \in A t}$ a.pp.cdc

Example 3 Let us consider the privacy preference described in Example 2 and let us further suppose that Charlotte also wants to specify a privacy preference for the electricity usage data. In particular, Charlotte wants to share her data with the Electricity Company and the Smart Home Company. Moreover, she wishes to: i) release the sensed data only for the administration of the electricity usage system, and ii) to join them with generic information for administration purpose, whereas iii) she does not pose any condition on what can't be derived from her data. Thus, she also specifies the following privacy preference: 〈electricityUsage, \{smart-home-company, electricity-company\}, $\langle\{$ admin $\}, \emptyset\rangle$, $\langle\{$ generic $\}, \emptyset,\langle\{$ admin $\}, \emptyset\rangle\rangle$, $\emptyset\rangle$.

Let us assume that the smart home network executes an equijoin on streams containing the electricity usage and temperature data generated by Charlotte's smart home system on $i d_{\text {room }}$ attributes, which we assume to have in both the two streams. Moreover, we assume that $i d_{\text {room }}$ has associated the same privacy preferences of temperature and electricity usage in both streams. The composed privacy preference for $i d_{\text {room }}$ in the stream containing temperature data is:

$\left\langle\right.$ id $_{\text {room }},\{$ smart-home-company $\},\langle\{$ admin $\}, \emptyset\rangle,\langle$ generic $\}, \emptyset$, $\langle\{$ admin $\}, \emptyset\rangle\rangle,\{$ sensitive $\}\rangle$.

This new privacy preference states that information on $i d_{\text {room }}$ can be released only for administration purpose to the Smart Home Company, can be joined with generic data for administration purpose, whereas sensitive information cannot be derived from this data.

\section{Decentralized privacy preference enforcement}

In order to assure that user privacy preferences are taken into consideration during data usage and processing, there is the need of an enforcement mechanism whose primary goal is to verify whether the privacy practices adopted by the entity wishing to consume the data, aka the consumer, satisfies data owners' privacy preferences. To make this compliance check automatic, we assume that consumer privacy practices are encoded into privacy policies, that is, a set of statements on how the consumer will use the users' data. Typically, a privacy policy specifies: the consumer identity (id), data attributes that consumer wishes to consume, purpose of the data usage, release of the data to third-parties and retention of the data in the consumer information system ${ }^{5}$ In what follows, we focus on the purpose and consumer id components of a privacy policy/preference and the related checks, in that they are the most important information used by the enforcement monitor. The enforcement mechanism has to verify whether the declared purpose of data usage and id of the consumer in the consumer's privacy policy is compliant with the privacy preferences specified by the users owning the smart object devices generating the data.

We handle compliance check through an aposteriori approach, that is, we perform compliance check only when data are going to be shared with consumers. The main motivation is that leaving compliance check to consumer SOs increases the possibility for data to be processed and thus to be eventually consumed according to user privacy preferences. For instance, let us consider the equi-join operation performed in Example 3 Let us assume that Charlotte's privacy preferences for electricity usage data only allow joint access with position data (e.g., coordinates of the individual), and let us also assume that joined streams have further attributes, in addition to electricity usage and temperature data. In such case, the equi-join operation in Example 3 is not compliant with Charlotte's privacy preferences. Performing a compliance check at this point would prevent to generate the joined stream, and further data flows would be cut. In contrast, by an aposteriori compliance check approach attributes in the joined streams can be further processed in the smart object network and consumers can consume the data that would not violate the privacy preferences of the users.

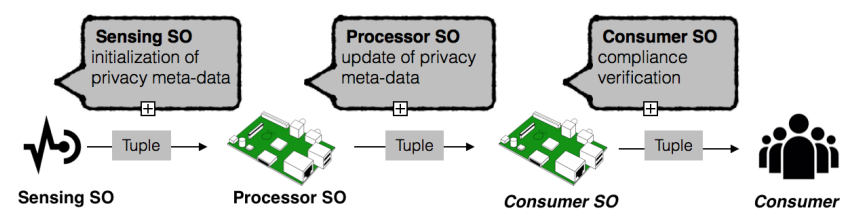

Figure 2: Privacy enforcement by SO roles

In order to push privacy preference enforcement at object level, we have to first complement the processed data with privacy meta-data that specify, for each piece of sensed or newly

\footnotetext{
${ }^{5}$ Privacy policies can be specified, as an example, by using XACML (https://docs.oasis-open.org/xacml/3.0/privacy/v1.0/xacml-3.0-privacyv1.0.html)
} 
created data, all information needed to enforce privacy preferences (see Figure 2). As an example, the sensing smart objects have to be able to associate with each piece of sensed data the corresponding privacy preferences specified by object owners. Moreover, processor SOs have to be able to locally generate additional privacy preferences for the newly created or modified data. This process requires to make processor SOs aware of how the new data have been created (e.g., which are the operations, the input data involved in the operations, as well as, the corresponding privacy preferences). Finally, smart objects playing the role of consumer SOs have to be able to locally perform compliance check.

\subsection{Privacy meta-data}

To model additional privacy information, we need to add, for each attribute contained into the original schema, three additional attributes, namely category, $p p$ (privacy preferences), and history.

Let us start discussing the category attribute. The category associated with an attribute is not a static information, as data associated with an attribute might be fused with other information, or simply modified, with the result of a change of its content and thus of its associated data categories. To cope with these dynamic aspects, we need to trace, for each attribute, its updated set of data categories, based on its current content. Secondly, in order to make a consumer SO able to perform privacy preference enforcement, it has to know the privacy preferences associated with each attribute. Such information is encoded in the additional $p p$ attribute. Moreover, information about every operation performed on data has to be documented alongside the data itself. Indeed, by design, compliance check is only performed by consumer SOs, just before data are sent to the consumer (see Figure 2). Processor SOs are only in charge of executing data processing and privacy meta-data generation, without caring about compliance checks. As such, it might be possible that in a smart object network, a processor SO performs an operation, e.g., a join, that breaks a jac or $c d c$ condition. In order to make a consumer SO able to verify if operations performed by all processor SOs satisfy jac and $c d c$ constraints, it has to know all operations performed on each piece of data (aka each attribute). At this purpose, we introduce, for each attribute in the stream schema, a new attribute, denoted as history, which contains a list of history entries, one for each operation performed on the corresponding attribute.

This privacy meta-data are directly encoded into the original data stream schema, obtaining thus a new schema, called Privacy-Enhanced Attribute Schema (PEAS), formally defined as follows:

Definition 3. (Privacy-Enhanced Attribute Schema). Let $S=\left(A_{1}, \ldots, A_{n}\right)$ be the schema of a data stream, where $A_{1}, \ldots, A_{n}$ are the data stream attributes. The PrivacyEnhanced Attribute Schema of $S$ is defined as $\bar{S}=\left(\bar{A}_{1}, \ldots, \bar{A}_{n}\right)$, such that $\bar{A}_{j}=\left(A_{j}\right.$, pp, category, history $), \forall j \in 1, \ldots, n$, where:

- $p p$ is the privacy preference associated with attribute $A_{j}$, as defined in Definition 1 .
- category is the set of data categories associated with the current value of attribute $A_{j}$;

- history is a list $\left\{H E_{1}, \ldots, H E_{m}\right\}$, containing an element for each operator $O p, A_{j}$ has undergone so far. $H E_{i}=\left(A C_{i}\right.$, $\left.R C_{i}\right)$, for $\forall i \in 1, \ldots, m$, where:

$-A C_{i}$ is the set of categories associated with attributes on which Op is executed for deriving $\bar{A}_{j}$;

$-R C_{j}$ is the set of data categories associated with $\bar{A}_{j}$ after the execution of $\mathrm{Op}$;

As depicted in Figure 2, privacy meta-data are initialized by sensing SOs, which collect the raw data and generate the corresponding PEAS schemas. In particular, at this stage in the PEAS schema the history field is initialized as empty, whereas the $p p$ and category fields contain privacy preferences and associated data category information defined by smart object owners. Processor SOs also modify attributes of PEAS schema. In particular, as it will be discussed in Section 4.2 , for each processed attribute, they have to update the category field, based on current value of the corresponding attribute as well as the history field.

Example 4 Let us refer again to Example 2, and, in particular, to attribute att containing the temperature data of Charlotte's smart home. Recalling the privacy preferences in Example 2, and supposing that the current temperature is 24.4 Celsius degree, the initial PEAS generated by the sensing SO, is the following:

- att $=\{24.4\}$,

- att.pp $=\langle$ temperature, $\{$ smart-home-company $\},\langle\{$ admin $\}$, $\emptyset\rangle,\langle\{$ generic, health $\}, \emptyset,\langle\{a d m i n\}, \emptyset\rangle\rangle,\{$ sensitive $\}\rangle$,

- att.category $=\{$ generic $\}$,

- att.history $=\{\emptyset\}$.

\subsection{PEAS generation}

For each sensed data, the sensing SO has to initialize the proper PEAS, with the privacy preferences specified by sensing SO's owner, the initial data category of the sensed data, and an empty history field. Additionally, after each operation, processor SOs should derive the privacy preferences of the eventually newly created attribute content, as well as update the category and history fields of each attribute involved in the operation. Hence, we assume that processor SOs are complemented with additional logic, implementing PEAS update.

Since PEAS generation in sensing SOs is quite straightforward, in that it mainly consists in initializing $p p$ and category attributes with predefined values, in the following we focus on PEAS update algorithms. PEAS update is mainly driven by the operations performed by processor SOs. As such, we define algorithms for all query operators except for the selection operator, as, by definition, this operator does not generate any new data, as such, no modification of the PEAS is needed. The algorithm receives as input the performed SQL operator $O p$ (e.g., avg, sum, $\times$, etc.) and input streams, used by the corresponding operation. In particular, we model an SQL operator based on operations it performs on the attributes. For example, the join 
of streams $S 1(a, b, c)$ and $S 2(a, d, e)$ on attribute $a$ is modelled as two parameters: \{join, (S1.a, S2.a)\}, that is, the involved attributes and the performed operation. Since, an SQL operator $O p$ might perform more than one operation (see, for instance, the $\Pi$ operator in the following Example 5), we formally define $O p$ as a set $\left\{\mathrm{Pa}_{1}, \ldots, \mathrm{Pa}_{l}\right\}$, where every $\mathrm{Pa}_{i}$ models a single operation. As such, $\mathrm{Pa}_{i}=\{$ Attributes, $f n\}, \forall i \in\{1, \ldots, l\}$, where Attributes is a set of attributes given as input to function $f n$ when operation $P a_{i}$ is executed.

Example 5 Suppose that the stream resulting from the equi-join operation illustrated in Example 3 also contains $h u$ midity data, thus resulting in S(temperature, electricityUsage, humidity). Let us assume to perform on this joined stream a projection $\Pi_{1}$ computing two values: the first obtained as multiplication of temperature and electricityUsage to estimate the number of people in the smart home ${ }^{6}$ and the second as execution of $f()$ that takes as input temperature and humidity and returns the air-quality information. $\Pi_{1}$ is thus modeled as: $\Pi_{1}=\left\{P a_{1}, P a_{2}\right\}$, where $P a_{1}=\{\{$ temperature, electricityUsage $\}$, $\times\}$ and $\mathrm{Pa}_{2}=\{\{$ temperature, humidity $\}, f()\}$.

PEAS generation algorithm exploits function derivePP(), that derives new composed privacy preferences, and function createHistoryEntry(), which creates a new history entry, based on the parameters of the performed operation. Finally, function derive $D C()$ determines the category of the new generated data given in input. To implement such a function, we assume the presence of a set of inference rules, called derivation paths. Each derivation path associates with a query operator and the set of categories of the data on which the operator is executed, the category of the data resulting from the operator execution. We therefore assume that derivation paths are present in every processor SO, so derived data category can be locally computed.

Example 6 Let us give an example of derivation path, by referring to the smart home scenario illustrated in Figure 1 and by assuming that also humidity information can be sensed. The derivation path $d p_{1}=\langle\{$ temperature,electricityUsage $\rangle, \times$, $\Pi$, sensitive $\rangle$, specifies that the knowledge of temperature and electricity usage allows, through the $\Pi$ and $\times$ operators, the derivation of the number of people inside the smart home. In contrast, the derivation path $d p_{2}=\langle\{$ temperature,humidity $\}$, $f(), \Pi$, air-quality $\rangle$ specifies that the knowledge of temperature and humidity usage allows derivation of air quality information, through operator $\Pi$ and function $f()$.

For PEAS derivation, Algorithm 1 takes as input tuples $s_{1}$ and $s_{2}$ (if operation is not $\bowtie$ then $s_{2}$ will be empty), and parameters modeling operation $\Pi, \Sigma$ or $\bowtie$. The algorithm returns an updated tuple, containing new attribute(s) generated by execution of the corresponding operation given in input and containing the updated pp, category and history fields associated with each attribute involved in the operation. In performing PEAS update for $\bowtie$ operation, attributes of tuples $s_{1}, s_{2}$ not used by $\bowtie$ remain unchanged, and by the nature of $\Sigma$ (such

\footnotetext{
${ }^{6}$ For simplicity, we assume that this is possible by multiplying electricity and
} temperature usage. as $\operatorname{avg}(), \operatorname{sum}()$, etc.), only one parameter will be present in Op.Parameters. The algorithm exploits previously discussed functions: derivePP() and createHistoryEntry().

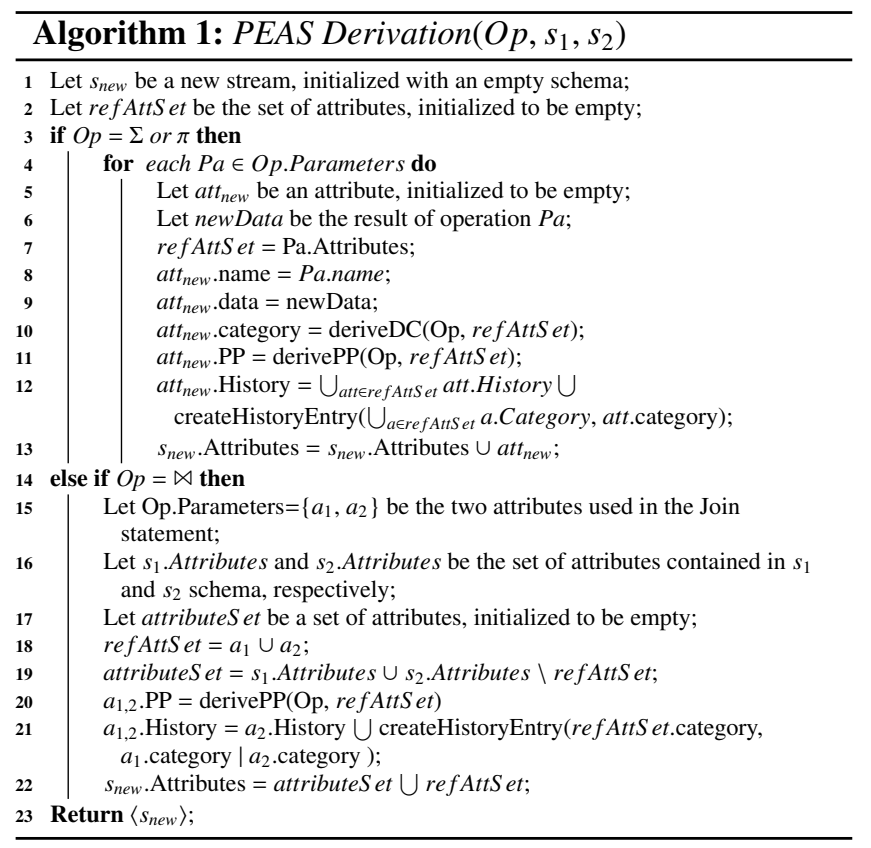

For $s_{\text {new }}$ and $a t t_{\text {new }}$, dot notation is used in accessing their elements specified by Definition 3

Example 7 Let us give an example of PEAS derivation by Algorithm 1 for the join of temperature and electricity usage data described in Example 3 Let us assume that the PEAS of electricity usage data has been created with the same logic given in Example 4. First, Algorithm 11 stores the parameters used in the join operation into refAttSet. All attributes of the two streams except parameters used in the join operation are stored as attributeSet to be included in the resulting stream without any modification. Then, the algorithm performs composition of privacy preferences for attributes in refAttSet and updates attributes in refAttSet with newly derived privacy preferences. Then, the new history entry newHE is created by function createHistoryEntry(), and added to the history fields of both the attributes used as parameters in the operation. Finally, the union of the attributes used as parameters of the operation and attributeSet are added to the resulting stream. The updated PEAS for the temperature attribute is therefore:

$-a t t=\{24.4\}$

- att.pp $=\langle$ temperature, $\{$ smart-home-company $\},\langle\{$ admin $\}$, $\emptyset\rangle,\langle\{$ generic $\}, \emptyset,\langle\{$ admin $\}, \emptyset\rangle\rangle,\{$ sensitive $\}\rangle$,

- att.category $=\{$ generic $\}$,

- att.history $=\{\{$ generic, generic $\},\{$ generic $\}\}$.

\subsection{Compliance verification}

Compliance verification is the operation of verifying if constraints specified by data privacy preferences are satisfied by the privacy policy of the consumer. These checks are performed by consumer SOs on every piece of data passing through them. More formally, a consumer SO receives as input a stream $S$ and returns as output a stream $\bar{S}$, containing only those tuples whose 
attributes' values satisfy privacy preferences of all the owners involved in the generation of attributes' contents. Compliance check implies three main steps: $(i)$ checking compliance of the consumer's identity (i.e., consumerID) with the allowed consumers (i.e., consumer) specified in the data privacy preference; (ii) checking compliance of the purpose (i.e., ap) of the consumer with the intended purposes (i.e., ip) specified in the data privacy preference; and (iii) checking compliance of the operations performed on the data (e.g., on each single attribute of the input stream) with constraints imposed by the joint access constraint jac and the category derivation constraint $c d c$.

Step $(i)$ is straightforward in that it should only be checked that consumerID specifies the identity of a consumer allowed to consume attribute $A$, that is, consumerID $\in$ A.pp.consumer. Regarding step (ii), this check is satisfied if ap specifies an allowed purpose, that is, a purpose contained in ip or in the set of allowed purposes implied by ip, if purposes are organized into a tree. More formally, denoting with $\overrightarrow{i p}$ the set of purposes implied by ip, ap complies with ip iff $a p \in A . p p . i \vec{p}$.

For step (iii), let us first discuss compliance of joint access constraint jac for a given attribute $A$ with privacy preference $p p$. Jac states which portions of user's personal data can be combined/aggregated with other data, and with which consumer purpose, as described in Section 3 The set of categories implied by jac denoted as $j \vec{a} c$, is composed of all categories that descend from the categories in jac.Adc which do not descend from any category of jac.Exc. We say that the jac of attribute $A$ is satisfied iff: ( $i$ ) all the accessed data categories in every history entry of $A$ belong to the set of categories implied by $j \vec{a} c$, and (ii) ap complies with the intended purpose component of jac. Formally:

Definition 4. Jac compliance. Let pp be a privacy preference specified for attribute A. Compliance of the pp.jac component is satisfied iff: ap $\in$ jac. $\vec{p} \wedge \wedge \forall H E \in$ A.history, $\forall c \in H E . \overrightarrow{A C} \rightarrow$ $c \in A . p p . j \vec{a} c$.

Let us now discuss compliance of category derivation constraint $c d c$ for a given attribute $A$ with privacy preference $p p$, which specifies the data categories that cannot be derived from the processing of attribute $A$. The set of data categories which on the basis of $c d c$ cannot be derived denoted as $c \vec{d} c$, is composed of all categories that specialize those referred to within $c d c$. Similarly to what happened for the jac component, history of the resulting attribute must be checked, since derivation of new attributes may be performed several times, and every derivation process has to comply with privacy preferences of the attribute. Therefore, we say that $c d c$ of attribute $A$ is satisfied iff all the data categories in every history entry of $A$ does not belong to the set of categories implied by $c \vec{d} c$. Formally:

Definition 5. Cdc compliance. Let pp be a privacy preferences specified for attribute A. Compliance of pp.cdc is satisfied iff: $\forall H E \in$ A.history, $\nexists c \in H E . R \overrightarrow{R C} \wedge c \in$ A.pp.c $\vec{d} c$.

Consumer SOs perform compliance check for any attribute in every tuple before sharing it with consumer. Let us present and describe our algorithm for checking compliance. As previously discussed, jac and $c d c$ compliance have to be ensured for every entry in the history field of PEAS. Algorithm 2 takes as input a tuple $s$, the id of the consumer consumerID, and the access purpose of the consumer ap. The algorithm returns an updated tuple, containing attribute(s) that complies with the privacy preferences of the involved users and access purpose of the consumer.

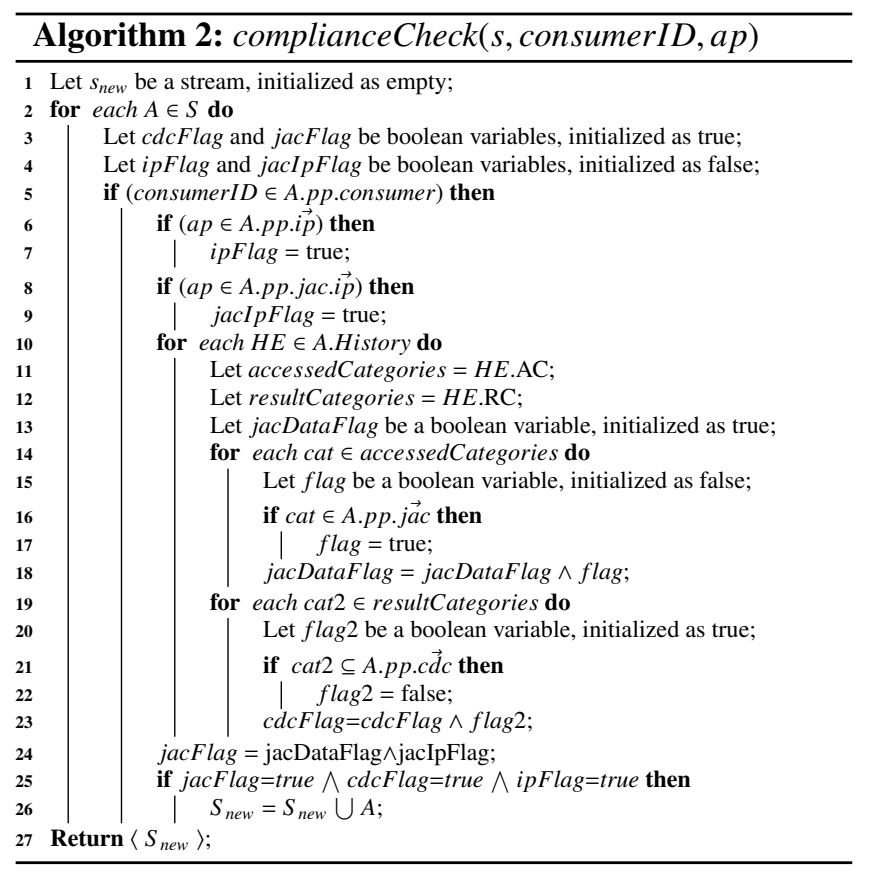

For $s_{\text {new }}$ and $a t t_{\text {new }}$, dot notation is used in accessing their elements specified by Definition 3

Example 8 Let us suppose that a processor SO performs the equi-join and projection $\Pi_{1}$ given in Example 7 . Moreover, suppose to have the derivation paths presented in Example 6 Algorithm 2 first checks compliance of the consumer (line 5). Let us assume that the consumer entity is smart-homecompany. In this case, the check returns true. Then, Algorithm 2 checks compliance of peopleCount.pp.ip and peopleCount.pp.jac.ip with the given consumer access purpose ap (lines 6 and 8). Let us assume that the consumer has specified admin as access purpose. In this case, the check returns true as both the purposes are admin, hence ipFlag and jacIpFlag are set to true. Then, for both jac and $c d c$, Algorithm 2 checks compliance of every history entry HE. Since peopleCount.history has two elements, the check is performed twice (line 10). In both of them, the algorithm checks that every category cat in HE.AC belongs to peopleCount.pp.jac (line 16). In our example, as peopleCount.pp.jac allows joint access with data belonging to category generic, jacDataFlag will remain true for both of the history entries of peopleCount.History. In the next step, the logical conjunction of jacDataFlag and jacIpFlag is done, to get jacFlag. Since both of them are true, jacFlag will be true. Similarly, the algorithm checks that every category cat 2 in HE.RC does not belong to peopleCount.pp.c $\vec{d} c$ (line 21). For the first history entry, $c d c F l a g$ will remain true, as category in 
$H E . R C$ is not sensitive. For the second history entry, $c d c F l a g$ will change to false, since the category in HE.RC is sensitive. Finally, since $c d c$ Flag is false (line 25), attribute peopleCount is not added to the final schema, hence it will not be shared with the consumer.

\section{Experiments}

In order to evaluate the overhead of the proposed mechanism, we have implemented several scenarios introduced in what follows.

\subsection{Experimental scenarios}

We developed four experimental scenarios to estimate the overhead at smart object and at network level.

\subsubsection{Processor Smart Objects I}

In this scenario, we consider processor SOs of second smartness level, able only to perform data selection and projection. In particular, we assume that processor SOs I are physically manufactured low capability devices, with hard-coded logic.To simulate this low capability devices, we have implemented smart objects using Freescale FRDM-K64 $\mathrm{F}^{7}$ with mbed operating system 8 This device has $256 \mathrm{~KB}$ RAM and ARM Cortex-M4 core. Coding has been done using $\mathrm{C}++$. Due to limited capabilities of the FRDM platform, we were not able to use real world data. As such, in this scenario, we used synthetic data, randomly created on the FRDM platform. We generate a tuple per second, containing a unique attribute of float data type, with randomly assigned value.

\subsubsection{Processor Smart Objects II}

In this scenario, we assume that the processor SO is a device with third smartness level, able to perform, in addition to selection and projection operations, also window-based operations, such as join and aggregation. This processor SO II has been implemented on a Raspberry Pi 3 Model B with Raspbian operating system, with 1GB RAM and a $1.2 \mathrm{GHz}$ 64-bit quadcore ARMv8 CPU. The implementation has been done with Java SE Development Kit 8u73. Moreover, to implement into processor SOs II an SQL engine able to manage window-based operations, we exploit Esper 9 We make use of the IoT-Lab testbec ${ }^{10}$ data, which provides a very large scale infrastructure suitable for testing small wireless sensor devices and heterogeneous communicating objects. Each node of IoT-Lab testbed produces tuples containing a unique attribute containing data sensed by that node. In particular, IoT-Lab provides three hardware platforms: WSN430, M3 Nodes, and A8 Nodes. Our experiments have been run with data transferred from M3 Nodes, where lumen level data, represented as float values, are sensed from light-to-digital sensor ISL29020 11

\footnotetext{
$\overline{{ }^{7} \text { developer.mbed.org/platforms/FRDM-K64F/ }}$

${ }^{8}$ www.mbed.com/en/platform/mbed-os/

${ }^{9}$ Www.espertech.com/esper

${ }^{10}$ www.iot-lab.info

${ }^{11}$ www.digikey.com/catalog/en/partgroup/is129020/14151
}

\subsubsection{Consumer Smart Objects}

We implemented a smart object with third smartness level, able to check privacy preference compliance. We adopt the same simulation and data generation strategies used for processor SOs II (see Section 5.1.2, where compliance verification algorithm has been implemented as an additional Java function.

\subsubsection{Smart Objects network}

We recall that the aim of this experiment is to measure the overall time overhead and bandwidth utilization implied by our enforcement mechanism on a network of smart objects. To simulate this scenario, we should implement a real network of smart objects, where each object is modified so as to embed the PEAS update algorithm as well as the compliance checks. However, the lack of standard platforms on which injecting our modified smart objects makes hard to deploy this setting. To overcome this issue, we decided to simulate a network of smart objects by leveraging on Complex Event Processing (CEP) systems [17]. We exploit the graph-based SQL modelling of CEP to simulate a network of objects, where every single operation in the CEP query is interpreted as a distinct smart object. As an example, the graph-based SQL query in Figure 3 can be interpreted as a network of two sensing SOs (i.e., the two INPUT operators), four processor SOs (i.e., the join, projection, aggregation and selection operators), and one final OUTPUT operator.

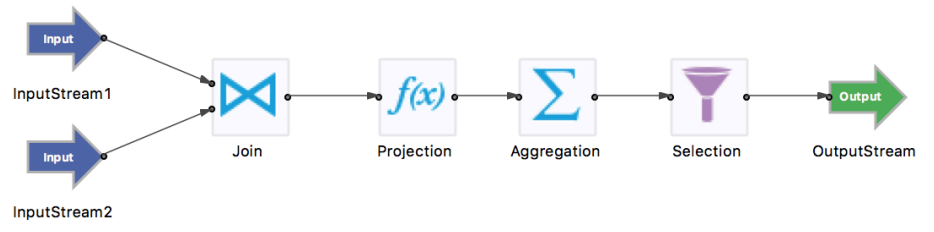

Figure 3: Graph-based SQL query

We adopted Streambase as CEP platform, as this product allows us to associate with an SQL operator an additional function, running a Java code, that will be executed by Streambase every time the operator processes an incoming tuple. As such, with Java SE Development Kit 8u73, we defined a set of functions implementing the PEAS update algorithms (cfr. Section 4). These functions have been properly associated with INPUT, Join, Selection, Projection, and Aggregation operators of Streambase. Following this design, the compliance verification algorithm should have been associated on the OUTPUT operator. However, since Streambase does not allow this, we implemented a new Streambase operator, to which the function implementing the compliance verification algorithm has been associated. This new defined operator is inserted just before the OUTPUT operator finalizing the query. We have exploited the feed simulation tool from Streambase Studio, that automatically generates and passes test data at specified rates to INPUT operators. Data rate has been fixed to 100 tuples per second for each stream. Number of attributes in each tuple has been regulated according to experiment settings (see discussion on query complexity in Section 5.2.1. 


\begin{tabular}{|c|c|c|c|c|}
\hline $\begin{array}{c}\text { Tested } \\
\text { dimensions }\end{array}$ & $\begin{array}{c}\text { Proc. } \\
\text { SO I }\end{array}$ & $\begin{array}{c}\text { Proc. } \\
\text { SO II }\end{array}$ & $\begin{array}{c}\text { Consumer } \\
\text { SO }\end{array}$ & $\begin{array}{c}\text { Smart } \\
\text { Object } \\
\text { network }\end{array}$ \\
\hline $\begin{array}{c}\text { Varying } \\
\text { complexity } \\
\text { of PP }\end{array}$ & $\checkmark$ & $\checkmark$ & $\checkmark$ & $\checkmark$ \\
\hline $\begin{array}{c}\text { Varying } \\
\text { query } \\
\text { complexity }\end{array}$ & $\checkmark$ & $\checkmark$ & $\checkmark$ & $\checkmark *$ \\
\hline $\begin{array}{c}\text { Varying } \\
\text { number of } \\
\text { sensing SOs }\end{array}$ & & $\checkmark$ & $\checkmark$ & \\
\hline
\end{tabular}

Table 2: Experiments: $\checkmark$ - time overhead, $\checkmark *$ time and bandwidth overhead

\subsection{Experimental results}

In executing our experiments, we considered three main characteristics that may impact the performance of the proposed solution. These are: privacy preferences complexity, queries complexity, and number of sensing smart objects. However, it was not possible to test all these dimensions in each scenario (see Table 5.2. Indeed, the experiment varying the number of sensing SOs requires to use IoT-Lab dataset, and, as discussed in Section 5.1, this dataset has been used only for processor SO II and consumer SO. Experiments have been executed by varying a single dimension and keeping fixed the others with worst case settings. Table presents a summary of conducted experiments. In the following, we illustrate the results.

\subsubsection{Varying the query complexity}

Processor SO I: these SOs are only able to perform $\sigma$ and $\Pi$ operators. Moreover, since $\sigma$ operator does not alter PEAS meta-data of processed attributes (cfr. Section 4), we only considered the П operator. We varied query complexity by increasing the number of attributes to be evaluated to perform the $\Pi$ operator. We recall that, according to the adopted notation, the $\Pi$ operator is modeled as a set of parameters, each one having a set of attributes to be evaluated. Thus, in this experiment, we have considered a $\Pi$ operator with a fixed number of parameters (i.e., 3), by varying the number of attributes in each of them. As an example, the simplest query is defined as a $\Pi$ operator with three parameters and two attributes for each parameter. Given a stream $\mathrm{S}(\mathrm{a}, \mathrm{b}, \mathrm{c})$, an example of simplest query is $\Pi_{(a+b),(c-b),(a \times c)}$. Execution times of queries with different complexity levels with and without the proposed privacy preference enforcement are illustrated in Figure 4. Even for the most complex queries, time overhead is less than $0.1 \mathrm{~ms}$ and performance overhead is less than $7 \%$.

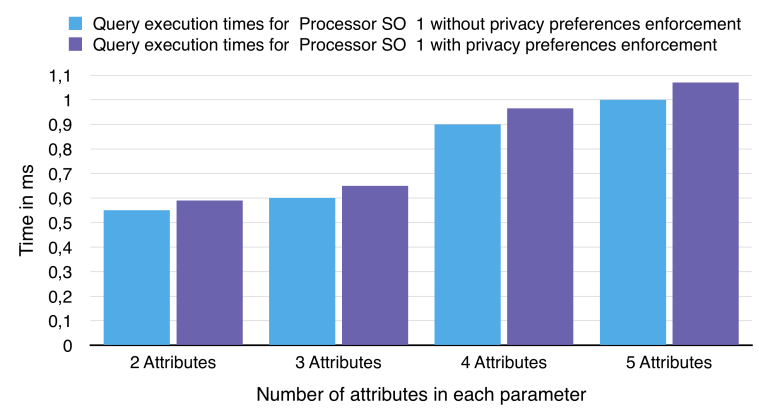

Figure 4: Varying the query complexity - Processor SO I
Processor SO II: query complexity can be estimated by the number of operators it contains. However, we have also to take into account that smart objects are constrained devices, so not being able to perform too complex SQL-like queries like those done by DBMSs. For instance, the processor SO II has been simulated on Raspberry Pi with only 1 GB RAM. To cope with this limitation, we have considered two sets of queries, with different complexity. The first set, named Simple Queries, contains queries consisting only of a $\Sigma$ operator. We select this aggregation operation as we expect it is the most common task performed by smart objects, that is, aggregating data sensed by sensors. The second set, named Complex Queries, contains queries performing a join of data sensed by two clusters of sensing SOs, then a projection over joined attributes, and, finally, an aggregation (i.e., average on 10 s window) on projected values. Again this type of queries represents a typical operation for smart objects. Table 3 shows query execution time with and without privacy preference enforcement. Even for the most complex queries, the time overhead is less than $0.71 \mathrm{~ms}$ and performance overhead is less than $9 \%$.

\begin{tabular}{|c|c|c|}
\hline $\begin{array}{c}\text { Processor } \\
\text { SO II }\end{array}$ & $\begin{array}{c}\text { Without } \\
\text { PP enforcement }\end{array}$ & $\begin{array}{c}\text { Proposed } \\
\text { mechanism }\end{array}$ \\
\hline $\begin{array}{c}\text { Simple } \\
\text { Queries }\end{array}$ & $5 \mathrm{~ms}$ & $5.36 \mathrm{~ms}$ \\
\hline $\begin{array}{c}\text { Complex } \\
\text { Queries }\end{array}$ & $8 \mathrm{~ms}$ & $8.71 \mathrm{~ms}$ \\
\hline
\end{tabular}

Table 3: Varying query complexity - Processor SO II

Consumer SO: even if consumer SOs are not designed to perform queries, but only compliance verification, we have to note that query complexity might impact the execution of this verification. Indeed, the more complex is the query, the more operations it contains. Thus, a complex query implies, as a consequence, more complex History fields to be evaluated by the compliance verification algorithm. As such, the aim of this experiment is to estimate how query complexity impacts the overhead given by compliance check. For this experiment, we used the same queries generated for processor SO II. Results of experiments are illustrated in Table 4. Even for complex queries, the time overhead is less than $0.44 \mathrm{~ms}$.

\begin{tabular}{|c|c|c|}
\hline Scenario & $\begin{array}{c}\text { Simple } \\
\text { queries }\end{array}$ & $\begin{array}{c}\text { Complex } \\
\text { queries }\end{array}$ \\
\hline Consumer $\mathrm{SO} \mathrm{II}$ & $0.18 \mathrm{~ms}$ & $0.44 \mathrm{~ms}$ \\
\hline
\end{tabular}

Table 4: Varying query complexity - Consumer SO

Smart Object network: We simulate a smart object network via a Streambase query, where each single Streambase operator acts as a smart object. Thus, increasing query complexity is like increasing the network complexity. In performing these experiments, we use a set of 10 queries with different numbers and types of operators. In particular, the simplest query, Q1, contains $1 \pi, 1 \sigma, 1 \bowtie$, and $1 \Sigma$ operator, whereas the most complex query, Q10, contains $10 \pi, 10 \sigma, 10 \bowtie$, and $10 \Sigma$ operators. Results of experiments are illustrated in Figure 5. As expected, less complex queries take less time to be processed. However, 


\begin{tabular}{|c|c|c|c|c|c|c|c|c|c|c|}
\hline Queries & Q1 & $\mathbf{Q 2}$ & $\mathbf{Q 3}$ & $\mathbf{Q 4}$ & $\mathbf{Q 5}$ & $\mathbf{Q 6}$ & $\mathbf{Q 7}$ & $\mathbf{Q 8}$ & Q9 & Q10 \\
\hline Query selectivity & $3,5 \%$ & $1,84 \%$ & $0,85 \%$ & $0,72 \%$ & $0,55 \%$ & $0,37 \%$ & $0,28 \%$ & $0,2 \%$ & $0,15 \%$ & $0,13 \%$ \\
\hline Extra bits per output tuple & 98 & 140 & 210 & 245 & 294 & 336 & 392 & 455 & 483 & 546 \\
\hline Bandwidth overload per hour & 3440 & 2576 & 1785 & 1764 & 1617 & 1243 & 1097 & 910 & 724 & 709 \\
\hline
\end{tabular}

Table 5: Varying query complexity - SO network - bandwidth overhead

even in complex network scenarios, the system overhead is always less than $10 \%$ which is less than $12 \mathrm{~ms}$.

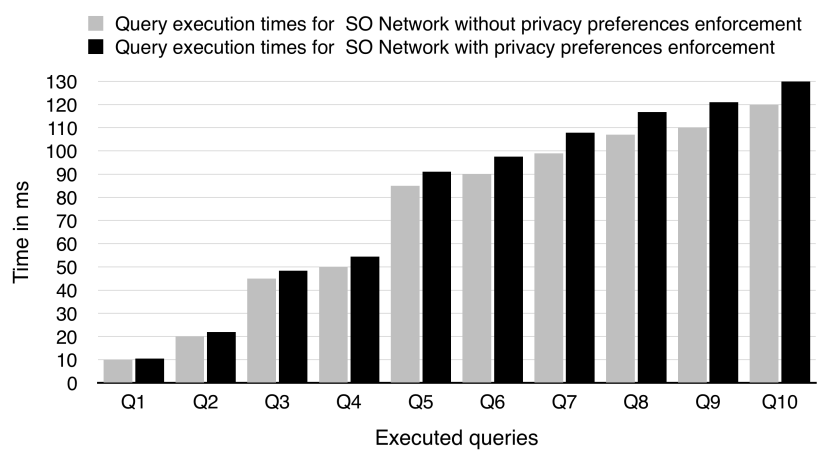

Figure 5: Varying query complexity - SO network - time overhead

Regarding bandwidth utilization, we have to note that the overhead is mainly implied by meta-data inserted in the original tuples. We recall that these meta-data are generated and updated by each single operator specified in the query. As such, we expect that a dimension impacting the bandwidth utilization is the query complexity. In addition to query complexity, another element that might impact this overhead is the query selectivity, that can be measured as the ratio of the number of tuples entering and number of tuples returned to/from a query. As query selectivity depends on characteristics of query operators (as an example, in the aggregation operator a bigger window size implies an higher query selectivity), we have modified the benchmark of 10 queries above described so as to tune the operators (e.g., window size in aggregation, selection conditions in $\Pi$ ) to obtain an increasing selectivity.

Number of bits added into output tuple after query execution are illustrated in the second line of Table 5. As expected, more complex queries increase the number of bits added to the metadata. However, it is relevant to note that even the most complex query consumes quite low bandwidth, $0.546 \mathrm{kbits}$, compared to the network capabilities of devices running similar queries (like Raspberry Pi) 12

In addition to that, we want to underline that we expect that complex queries will have higher selectivity over input tuples (see first line of Table 5), thus less output tuples will be generated. Thus, on the one hand a more complex query increases the number of added bits, but on the other hand, it decreases the number of output tuples and thus the overall bandwidth utilization. To better explain this balance, we run an experiment simulating a smart home scenario generating 1,000 tuples per hour, each containing a float data type attribute (e.g., room temperature, used electricity in $\mathrm{kw}$ ). This implies 32 bits in each single

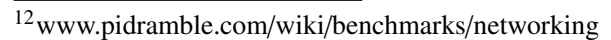

tuple. In case that no queries are executed, that is, all tuples are flooding the network, we have $32 \mathrm{kbit}$ overall bandwidth per hour. From the third line of Table 5, we can see that the overall overhead decreases by varying query complexity, aka query selectivity. Therefore, even in the worst case, output tuples generated by queries consume quite low bandwidth, i.e. 3.4 kbits.

\subsubsection{Varying complexity of privacy preferences}

We recall that a privacy preference poses a set of conditions to be verified on consumer privacy policy, namely ip, jac, and $c d c$ (see Section 3). The presence of these conditions implies checks and operations both in the compliance verification algorithm as well as in the PEAS generation algorithms. As such, we can see the number of conditions specified in a given $p p$ as a dimension to measure $p p$ complexity. In view of this, we generated two sets of privacy preferences with two different levels of complexity. In particular, privacy preferences in the first set, named Simple PP, have been defined as very simple preferences with a unique condition on the intended purpose field, and thus their compliance verification is easy to be performed. In contrast, privacy preferences in the second set, named Full PP, have been defined with a condition on each field (i.e., ip, jac, $c d c$ ), so as to make harder the compliance verification. More precisely, privacy preferences have been defined in such a way that their constraints for both Simple PP and Full PP refer to random elements of the data category and purpose trees, which, in turn, have been populated with synthetic data. In particular, we have considered 100 elements in both the data category and the purpose trees. This test case has been applied to all experiment scenarios, as described in the following.

Processor SO I: since FRDM is an hard-coded device with limited capabilities, we assumed that with each attribute in the synthetic dataset the same privacy preference is associated (i.e., every attribute has the same $p p$ value). This corresponds to a scenario where the implemented processor SO I receives as input a flow of data generated by a sensing SO owned by a single user, as such all data are marked with the same privacy preference. However, to test how complexity of privacy preferences impacts the local overhead of processor SO I, we run several times these experiments, changing in each execution the privacy preferences associated with attributes, by selecting them both from Simple PP and Full PP sets.

Processor SO II, Consumer SO, and Smart Object network: differently from processor SOs I, privacy preferences are dynamically generated and randomly assigned to each attribute of the tuples entering in the considered smart object.

In performing these experiments we adopt the settings shown in Table 6, which also presents the execution time of queries without privacy preferences (i.e., No $P P$ ), with simple privacy preferences (Simple $P P$ ), and complex privacy preferences $($ Full $P P)$. As expected, simple privacy preferences imply less 


\begin{tabular}{|c|c|c|c|c|}
\hline Scenario & Settings & $\begin{array}{c}\text { No } \\
\text { PP }\end{array}$ & $\begin{array}{c}\text { Simple } \\
\text { PP }\end{array}$ & $\begin{array}{c}\text { Full } \\
\text { PP }\end{array}$ \\
\hline $\begin{array}{c}\text { Processor } \\
\text { SO I }\end{array}$ & Complex queries & $0.31 \mathrm{~ms}$ & $0.325 \mathrm{~ms}$ & $0.34 \mathrm{~ms}$ \\
\hline $\begin{array}{c}\text { Processor } \\
\text { SO II }\end{array}$ & $\begin{array}{c}\text { Complex queries, } \\
10 \text { sensing SOs } \\
100 \% \text { assoc. PP }\end{array}$ & $3 \mathrm{~ms}$ & $3.10 \mathrm{~ms}$ & $3.28 \mathrm{~ms}$ \\
\hline Consumer SO & $\begin{array}{c}\text { Complex queries, } \\
10 \text { sensing SOs } \\
100 \% \text { assoc. PP }\end{array}$ & $\emptyset$ & $0.08 \mathrm{~ms}$ & $0.34 \mathrm{~ms}$ \\
\hline SO network & Complex queries & $10 \mathrm{~ms}$ & $10.74 \mathrm{~ms}$ & $10.99 \mathrm{~ms}$ \\
\hline
\end{tabular}

Table 6: Overhead by varying PP complexity

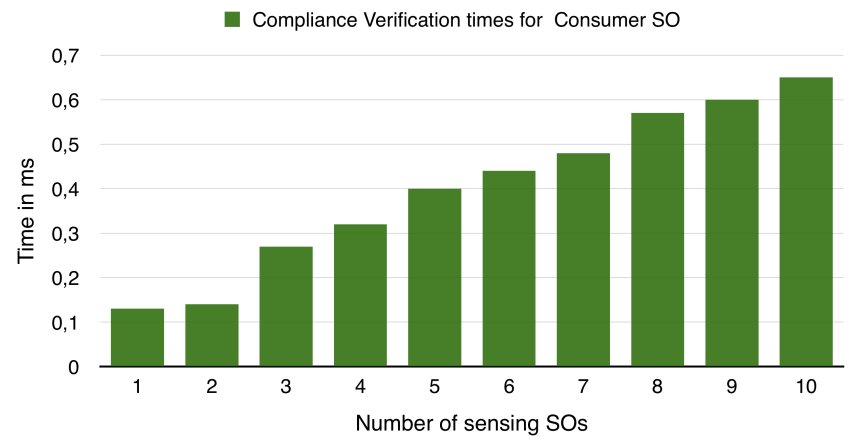

Figure 7: Varying the number of sensing SOs in Consumer SO scenario

As expected, for both scenarios having more nodes in the system takes more time to process. ${ }^{13}$ For processor SO II, even in the worst case where we have 10 sensing SOs, overhead is under $0.7 \mathrm{~ms}$, thus performance overhead is less than $9 \%$. Compliance check performed by consumer SOs always takes less than $0.7 \mathrm{~ms}$.

\section{Related Work}

In recent years, security and privacy in the IoT domain have been deeply investigated, with the results that various approaches have been proposed for dealing with different aspects of security as well as of privacy. In this section, we provide an overview of those proposals that are more related to the proposed framework. In particular, we focus on those approaches that enforce, in some ways, users' privacy. However, we also have to note that literature offers several interesting proposals that, like our framework, deal with the problem of decentralized policy enforcement. All these efforts have been done in domains different from IoT, but they deserve to be cited and compared to our solution. In the following, we summarize works in these two directions.

\subsection{Enforcement of users' privacy in the IoT domain}

So far different access control models have been exploited in the IoT domain: role based access control (RBAC) (e.g., [4], [18]); capability based access control (CapBAC) (e.g., [2]); attribute based access control (ABAC) (e.g., [3], [19]), and access control models based on semantic rules (e.g., [20]). Although these proposals are instrumental to control how users' personal data are used, and thus, in some sense, to protect users' privacy, they do not make users able to provide their own preferences on how their data have to be used and distributed. In contrast, our proposal makes user able to have a full control on how data have to be processed (e.g., accessed, aggregated, released).

User's privacy preferences have been considered in [21], which proposes a framework avoiding inference of personal data due to data fusion. Users specify their privacy preferences in terms of a level of confidentiality associated with each data.

\footnotetext{
${ }^{13}$ We note that, we have performed similar experiments by varying the percentage of sensing SOs with an associated privacy preferences, and we have observed same overhead trends.
} 
The proposed framework consists of a central unit, called Personal Data Manager (PDM), that manages personal data collected by different devices, playing thus the role of a gateway between users and third party applications. A further module, called Adaptive Interface Discovery Service (AID-S), computes the risk of inference associated with a data disclosure, via probabilistic models and learning algorithms (e.g., RST, KNN, Bayes Filter, HMM etc.). Based on this risk value, AID-S recommends optimal privacy settings to users to reduce the privacy risks. Similar to our proposal, also this approach considers user's perspective, but only in stating the confidentiality level of personal data, whereas our privacy model considers several dimensions of a privacy preference. Additionally, we enforce privacy of the user against data inferences in a decentralized setting, being able to pose more limitations on possible data fusions.

Compliance of user's privacy preferences with third party's privacy policies have been considered in [22]. Here, it has been proposed an application for mobile phones that supports customers in making privacy decisions. Privacy preferences are automatically generated according to the result of a questionnaire filled by users. The application informs the user whether his/her privacy preferences complies with the corporate's privacy policies. In contrast, we handle privacy in a bigger application scenario, that is, we enforce user privacy preferences in a decentralized IoT scenario, where different smart objects may apply their own queries over data and other parties may get involved in data processing.

Similar to our approach, other proposals have targeted smart environments (e.g., smart home and smart city systems) with the aim of protecting users' privacy. In [23], authors address the security and privacy problems of IoT smart home at the network level, that is, by monitoring network activities of IoT devices to detect suspicious behaviors. An external entity, called Security Management Provider (SMP) has been proposed. SMP can add access control rules to protect specific IoT devices or can apply dynamic policies to change access control rules depending on the context (e.g., the family members being present or absent from the house). This proposals aims at protecting privacy of the user by limiting access on data through an external entity, i.e., SMP, with the use of context information. In contrast, in our approach, we enforce user-defined privacy preferences to protect users' privacy in a decentralized scenario.

In [24], a two layered architecture is proposed for protecting users' privacy in smart city applications. A trusted layer is designed to store real identities of individuals that can be processed only by the platform's components, without disclosing the identities to the outside world. In contrast, an untrusted second layer only makes generic, unidentifiable and identity-independent information available to external applications. Even if this proposal protects personal data, this is enforced only inside the trusted layer, without considering future operations that may be done on the released data to infer new sensitive information. Moreover, users are not able to set and enforce their own privacy preferences.

\subsection{Decentralized policy enforcement}

A notable example of decentralized privacy management is represented by the sticky policy approach [25]. According to this approach user privacy preferences are strictly associated (sticky) with users' data. [25] describes the core mechanisms required for managing sticky policies, along with Public Key Infrastructure (PKI) and encryption methodologies to attach sticky policies with data as well as to enforce them. [26] presents a distributed enforcement approach for sticky policies that permits data to be disseminated across heterogeneous hardware and software environments without pre-existing trust relationships. Also, [27] presents a sticky policy approach to manage the access to IoT resources by allowing users to set and manage access control policies on their own data. In this approach, sticky policies allow to define: owner of the data; purposes for which the data can be used; a timestamp that points out the validity; and constraints which represent the rules for filtering the data with obligations and restrictions.

Our solution has some similarities with the sticky policy approach, as we share the same goal, that is, decentralized enforcement of user privacy preferences. However, in our proposal we go beyond the traditional privacy preference model, where constrains are posed mainly on purpose, retention time, and third party usage by proposing a mechanism to derive privacy preferences for newly generated data.

Additionally, sticky policy approaches use encryption mechanisms to enhance privacy, whereas in our approach encryption is just used to secure communication. Indeed, encryption mechanisms add extra level of complexity and demand higher resources from the devices. However, we have to note that cryptographic solutions have been often used to enforce distributed access control in several domains, like online social networks (e.g., [28, 29]), or cloud infrastructure (e.g., [30, 31]). In the following we focus on efforts done in wireless sensor networks, as these are more relevant for the IoT domain. For instance, [32] presents a distributed privacy preserving access control scheme designed for network of sensor nodes owned by different users, connected via a network, and managed via an offline certificate authority. In the proposed scheme, access control is regulated exploiting tokens that users have to pre-buy from the network owner before entering the sensor network. Users can query sensor data with unspent tokens and sensor nodes are subject to validate the token and grant appropriate amount of requested data. The scheme makes use of blind signatures in token generation, which ensures that tokens are publicly verifiable yet they are unlikable to user identities. Similarly to the previous cited work, [5] presents a distributed protocol for achieving privacypreserving access control for sensor networks by exploiting a ring signature scheme. To query sensor nodes, a user needs to build a ring signature along with the query command and send them together to sensor nodes. However such approaches do not consider privacy preferences of the data owner.

Since one of the features of the proposed framework is to derive privacy preferences for new data, we have to note that literature offers also works dealing with composition of heterogeneous access control policies. These have been done with the 
goal of composing a set of access control policies into a single one [33, 34]. For instance, [33] proposes a semantic framework for policy composition without committing to a specific access control model. Access control policies are modelled as four valued predicates. Similarly, [34] proposes an algebra for constructing security policy from different policies.

\section{Conclusion}

In this paper, we proposed a decentralized privacy enforcement mechanism for IoT, where compliance check of user individual privacy preferences is performed directly by smart objects. The experimental evaluation has shown a negligible time and bandwidth overhead.

We plan to extend our proposal according to several directions. We recall that the proposed decentralized privacy enforcement mechanism assumes the existence of a set of "derivation paths", that is, a set of inference rules able to predict the data category resulting from the execution of an operator. As a first future work, we aim at investigating how to derive these rules. At this purpose, we plan to exploit existing data fusion schemes proposed for IoT domain [35, 36], where different methodologies have been designed (e.g., probability based, artificial intelligence based) aiming at combining data from multiple sensors to produce more accurate, more complete, and more dependable information that could not be possible to achieve through a single sensor. These methods could be deployed in our framework to model the data flow, and thus derivation paths. Specifically, multi-dimensional data fusion algorithms, such as [36, 35], could be used to extract association rules from data generated by subnet of sensing SOs. Second, we plan to implement a hybrid approach for compliance check, that combines apriori and aposteriori compliance verification, to decrease the performance overhead of the system.

We also plan to improve usability of the system. Indeed, we are aware that an average user of an IoT application may not be skilled enough to understand potential privacy threats and to properly set up his/her privacy preferences. At this purpose, we plan to investigate tools to help users in setting their privacy preferences, based on learning from their habits, as it has been previously done in other domains (e.g., Online Social Networks [37]).

\section{References}

[1] J. Gubbi, R. Buyya, S. Marusic, M. Palaniswami, Internet of things (iot): A vision, architectural elements, and future directions, Future Generation Computer Systems 29 (2013) 1645-1660.

[2] A. F. Skarmeta, J. L. Hernández-Ramos, M. V. Moreno, A decentralized approach for security and privacy challenges in the internet of things, in: Internet of Things (WF-IoT), 2014 IEEE World Forum on.

[3] S. Sicari, A. Rizzardi, D. Miorandi, C. Cappiello, A. Coen-Porisini, Security policy enforcement for networked smart objects, Computer Networks 108 (2016) $133-147$

[4] J. Liu, Y. Xiao, C. P. Chen, Authentication and access control in the internet of things, in: Distributed Computing Systems Workshops (ICDCSW), 2012 32nd International Conference on, IEEE, pp. 588-592.

[5] D. He, J. Bu, S. Zhu, S. Chan, C. Chen, Distributed access control with privacy support in wireless sensor networks, IEEE Transactions on Wireless Communications 10 (2011) 3472-3481.
[6] B. Carminati, P. Colombo, E. Ferrari, G. Sagirlar, Enhancing user control on personal data usage in internet of things ecosystems, in: Services Computing (SCC), 2016 IEEE International Conference on, IEEE, pp. 291-298.

[7] K. Yasumoto, H. Yamaguchi, H. Shigeno, Survey of real-time processing technologies of IoT data streams, Journal of Information Processing (2016).

[8] J. Voas, Networks of things, NIST SPECIAL PUBLICATIONS (SP 800) (2016).

[9] ITU-T, 2060, Overview of Internet of Things (2012).

[10] O. Arias, J. Wurm, K. Hoang, Y. Jin, Privacy and security in internet of things and wearable devices, IEEE Transactions on Multi-Scale Computing Systems 1 (2015) 99-109.

[11] S. Saad, I. Traore, A. Ghorbani, B. Sayed, D. Zhao, W. Lu, J. Felix, P. Hakimian, Detecting p2p botnets through network behavior analysis and machine learning, in: Privacy, Security and Trust (PST), 2011 Ninth Annual International Conference on, IEEE, pp. 174-180.

[12] C. Liu, J. Yang, Y. Zhang, R. Chen, J. Zeng, Research on immunity-based intrusion detection technology for the internet of things, in: Natural Computation (ICNC), 2011 Seventh International Conference on, volume 1, IEEE, pp. 212-216.

[13] J. Zhen, J. Li, M. J. Lee, M. Anshel, A lightweight encryption and authentication scheme for wireless sensor networks, International Journal of Security and Networks 1 (2006) 138-146.

[14] Q. Wen, X. Dong, R. Zhang, Application of dynamic variable cipher security certificate in internet of things, in: Cloud Computing and Intelligent Systems (CCIS), 2012 IEEE 2nd International Conference on, volume 3, IEEE, pp. 1062-1066.

[15] T. Heer, S. Götz, O. G. Morchon, K. Wehrle, Alpha: an adaptive and lightweight protocol for hop-by-hop authentication, in: Proceedings of the 2008 ACM CoNEXT Conference, ACM, p. 23.

[16] M. Conti, R. Di Pietro, L. Mancini, A. Mei, Distributed detection of clone attacks in wireless sensor networks, IEEE transactions on dependable and secure computing 8 (2011) 685-698.

[17] E. Wu, Y. Diao, S. Rizvi, High-performance complex event processing over streams, in: Proceedings of the 2006 ACM SIGMOD, ACM.

[18] E. Barka, S. S. Mathew, Y. Atif, Securing the web of things with rolebased access control, in: International Conference on Codes, Cryptology, and Information Security, Springer, pp. 14-26.

[19] N. Ye, Y. Zhu, R.-c. Wang, Q.-m. Lin, An efficient authentication and access control scheme for perception layer of internet of things (2014).

[20] A. H. Celdrán, F. J. G. Clemente, M. G. Pérez, G. M. Pérez, Secoman: A semantic-aware policy framework for developing privacy-preserving and context-aware smart applications, IEEE Systems Journal (2016).

[21] I. Torre, F. Koceva, O. R. Sanchez, G. Adorni, A framework for personal data protection in the iot, in: Internet Technology and Secured Transactions (ICITST), 2016 11th International Conference for, IEEE, pp. 384 391.

[22] G. Broenink, J.-H. Hoepman, C. v. Hof, R. Van Kranenburg, D. Smits, T. Wisman, The privacy coach: Supporting customer privacy in the internet of things, arXiv preprint arXiv:1001.4459 (2010).

[23] V. Sivaraman, H. H. Gharakheili, A. Vishwanath, R. Boreli, O. Mehani, Network-level security and privacy control for smart-home iot devices, in: WiMob, IEEE, pp. 163-167.

[24] O. Mazhelis, A. Hämäläinen, T. Asp, P. Tyrväinen, Towards enabling privacy preserving smart city apps, in: Smart Cities Conference (ISC2), 2016 IEEE International, IEEE, pp. 1-7.

[25] S. Pearson, M. Casassa-Mont, Sticky policies: an approach for managing privacy across multiple parties, Computer (2011).

[26] J. Brown, D. M. Blough, Distributed enforcement of sticky policies with flexible trust, in: 2015 IEEE 7th International Symposium on Cyberspace Safety and Security (CSS), IEEE, pp. 1202-1209.

[27] S. Sicari, A. Rizzardi, D. Miorandi, A. Coen-Porisini, Security towards the edge: Sticky policy enforcement for networked smart objects, Information Systems 71 (2017) 78-89.

[28] B. Carminati, E. Ferrari, A. Perego, Enforcing access control in webbased social networks, ACM Transactions on Information and System Security (TISSEC) 13 (2009) 6.

[29] S. Jahid, P. Mittal, N. Borisov, Easier: Encryption-based access control in social networks with efficient revocation, in: Proceedings of the 6th ACM Symposium on Information, Computer and Communications Secu- 
rity, ACM, pp. 411-415.

[30] G. Wang, Q. Liu, J. Wu, Hierarchical attribute-based encryption for finegrained access control in cloud storage services, in: Proceedings of the 17th ACM conference on Computer and communications security, ACM, pp. 735-737.

[31] S. Ruj, M. Stojmenovic, A. Nayak, Decentralized access control with anonymous authentication of data stored in clouds, IEEE transactions on parallel and distributed systems 25 (2014) 384-394.

[32] R. Zhang, Y. Zhang, K. Ren, Distributed privacy-preserving access control in sensor networks, IEEE Transactions on Parallel and Distributed Systems 23 (2012) 1427-1438.

[33] G. Bruns, D. S. Dantas, M. Huth, A simple and expressive semantic framework for policy composition in access control, in: Proceedings of the 2007 ACM workshop on Formal methods in security engineering, ACM, pp. 12-21.

[34] P. Bonatti, S. De Capitani di Vimercati, P. Samarati, An algebra for composing access control policies, ACM Transactions on Information and System Security (TISSEC) 5 (2002) 1-35.

[35] J. Zhou, L. Hu, F. Wang, H. Lu, K. Zhao, An efficient multidimensional fusion algorithm for iot data based on partitioning, tsinghua science and technology 18 (2013) 369-378.

[36] A. Ahmad, A. Paul, M. Rathore, H. Chang, An efficient multidimensional big data fusion approach in machine-to-machine communication, ACM Transactions on Embedded Computing Systems (TECS) 15 (2016) 39.

[37] L. Fang, K. LeFevre, Privacy wizards for social networking sites, in: Proceedings of the 19th international conference on World wide web, ACM, pp. 351-360. 